\title{
DISCOMFORT IN ACADEMIA AND ITS EFFECT ON READINESS FOR CHANGE
}

Laurie A. Branch, Tony Lingham, and Kalle Lyytinen

The BRC Academy Journal of Business 8, no. 1 (2019): 29-59.

https://dx.doi.org/10.15239/ j.brcacadje.2020.08.01.ja02

\section{WEB APPENDIX}

https://dx.doi.org/10.15239/ j.brcacadje.2020.08.01.wa02 


\section{TABLE 1}

\section{Example of Items, EFA, Correlations, and Reliabilities for Discomfort as a Factor ${ }^{1}$}

\section{CONSTRUCT: DISCOMFORT (Reflective) Example of Items By Factor}

Workload Impact (WL): My institution typically expects me to work harder when we are in the midst of a change.

Job Security Impact (JS):Changes at work are likely to impact my job security.

Relationship Impact (R): I have found that changes at my institution typically lead to changes in my relationships at work.

Emotional Impact (EMO): I am frustrated when my institution asks me to do extra work because we are making a change.

Work Focus Impact (WF): It is hard to stick to my plans at work when my institution is making changes.

\begin{tabular}{|c|c|c|c|c|c|}
\hline FACTOR & $\begin{array}{l}\text { Work Load } \\
\text { Impact }\end{array}$ & $\begin{array}{c}\text { Job Security } \\
\text { Impact }\end{array}$ & $\begin{array}{l}\text { Relationship } \\
\text { Impact }\end{array}$ & $\begin{array}{l}\text { Emotional } \\
\text { Impact }\end{array}$ & $\begin{array}{l}\text { Work Focus } \\
\text { Impact }\end{array}$ \\
\hline Abbreviation & $\mathbf{W L}$ & $\mathbf{J S}$ & $\mathbf{R}$ & EMO & WF \\
\hline Avg. Loading & .762 & .843 & .856 & .700 & .737 \\
\hline $\mathrm{R} 1$ & & & .862 & & \\
\hline $\mathrm{R} 2$ & & & .897 & & \\
\hline R3 & & & .810 & & \\
\hline JSEC1 & & .949 & & & \\
\hline JSEC2 & & .676 & & & \\
\hline JSEC3 & & .905 & & & \\
\hline EMO1 & & & & .862 & \\
\hline EMO2 & & & & .811 & \\
\hline EMO3 & & & & .565 & .226 \\
\hline EMO4 & & & & .561 & \\
\hline WL1 & .680 & & & & \\
\hline WL2 & .775 & & & & \\
\hline WL3 & .832 & & & & \\
\hline WF1 & & & & & .709 \\
\hline WF2 & & & & & .729 \\
\hline WF3 & & & & & .773 \\
\hline \multicolumn{6}{|c|}{ CORRELATIONS } \\
\hline $\mathrm{WL}$ & $0.822^{\mathrm{a}}$ & & & & \\
\hline JS & 0.133 & 0.874 & & & \\
\hline $\mathrm{R}$ & 0.348 & 0.271 & 0.890 & & \\
\hline EMO & 0.292 & 0.229 & 0.108 & 0.806 & \\
\hline WF & 0.486 & 0.319 & 0.352 & 0.616 & 0.807 \\
\hline
\end{tabular}

\footnotetext{
${ }^{1}$ For our EFA analysis we used Maximum Likelihood extraction and Promax rotation with a maximum of 25 iterations for conversion. We forced an 8 factor solution comprised of the 5 factors shown here plus self-efficacy (SE1-8), perceived collective efficacy (PCE1-8), and intentional readiness for change (RC1-3). We suppressed values below .20. The sample size was 435 .
} 
TABLE 2

Demographical Information of Sample

\begin{tabular}{|c|c|c|c|c|}
\hline Demographic & Grouping Variable & $\begin{array}{c}\text { \# of } \\
\text { Respondents } \\
\text { (out of 489) }\end{array}$ & $\begin{array}{l}\% \text { of Respondents } \\
\text { (out of } 100 \% \text { ) }\end{array}$ & $\begin{array}{l}\text { Accredited Business } \\
\text { School Population* } \\
\text { (out of } 100 \%)\end{array}$ \\
\hline Gender & Male/ Female & $335 ; 154$ & $69 \% ; 31 \%$ & $70 \% ; 30 \%$ (a) \\
\hline Tenure & $\begin{array}{l}\text { Tenured/Tenure } \\
\text { Track/Non-Tenure Track }\end{array}$ & $329 ; 92 ; 68$ & $67 \% ; 19 \% ; 14 \%$ & $56 \% ; 23 \% ; 20 \%(b)$ \\
\hline Professorial Rank & $\begin{array}{l}\text { Full/Asst/Assoc } \\
\text { Lecturer/Instructor/Other }\end{array}$ & $\begin{array}{c}219 / 101 / 140 \\
12 / 3 / 14 \\
\end{array}$ & $\begin{array}{c}45 \% ; 21 \% ; 29 \% \\
2 \% ; 1 \% ; 3 \% \\
\end{array}$ & $\begin{array}{l}33 \% ; 25 \% ; 27 \%(\mathrm{c}) \\
\text { na; } 15 \%(\mathrm{c}) ; \text { na }\end{array}$ \\
\hline $\begin{array}{l}\text { Organization } \\
\text { Type }\end{array}$ & $\begin{array}{l}\text { Public NFP/Private NFP/ } \\
\text { FP }\end{array}$ & $258 ; 227 ; 4$ & $53 \% ; 46 \% ; 1 \%$ & $57 \% ; 43 \%$ (a) \\
\hline $\begin{array}{l}\text { U.S. Business } \\
\text { Schools Ranking }\end{array}$ & $\begin{array}{l}\text { Top } 20 / 1^{\text {st }} \text { and } 60^{\text {th }} / \text { ranked } \\
\text { Not ranked/I don't know }\end{array}$ & $\begin{array}{l}37 ; 140 ; 147 \\
86 ; 79\end{array}$ & $\begin{array}{c}8 \% ; 29 \% ; 30 \% \\
18 \% ; 16 \%\end{array}$ & \\
\hline
\end{tabular}

*Source: a) Business School Data Trends (2012) AACSB; http://www.aacsb.edu/publications/businesseducation/2012-Data-Trends.pdf b) Business School Data Trends (2011) AACSB; http://www.aacsb.edu/publications/businesseducation/2011-data-trends.pdf

c) Faculty Profile (2011-12) AACSB: graciously provided by AACSB Knowledge Services in private correspondence

TABLE 3

Validity and Reliability of our CFA, Model Fit for CFA, Correlations and Reliabilities after Removing SE1 and SE7

Validity and Reliability of CFA

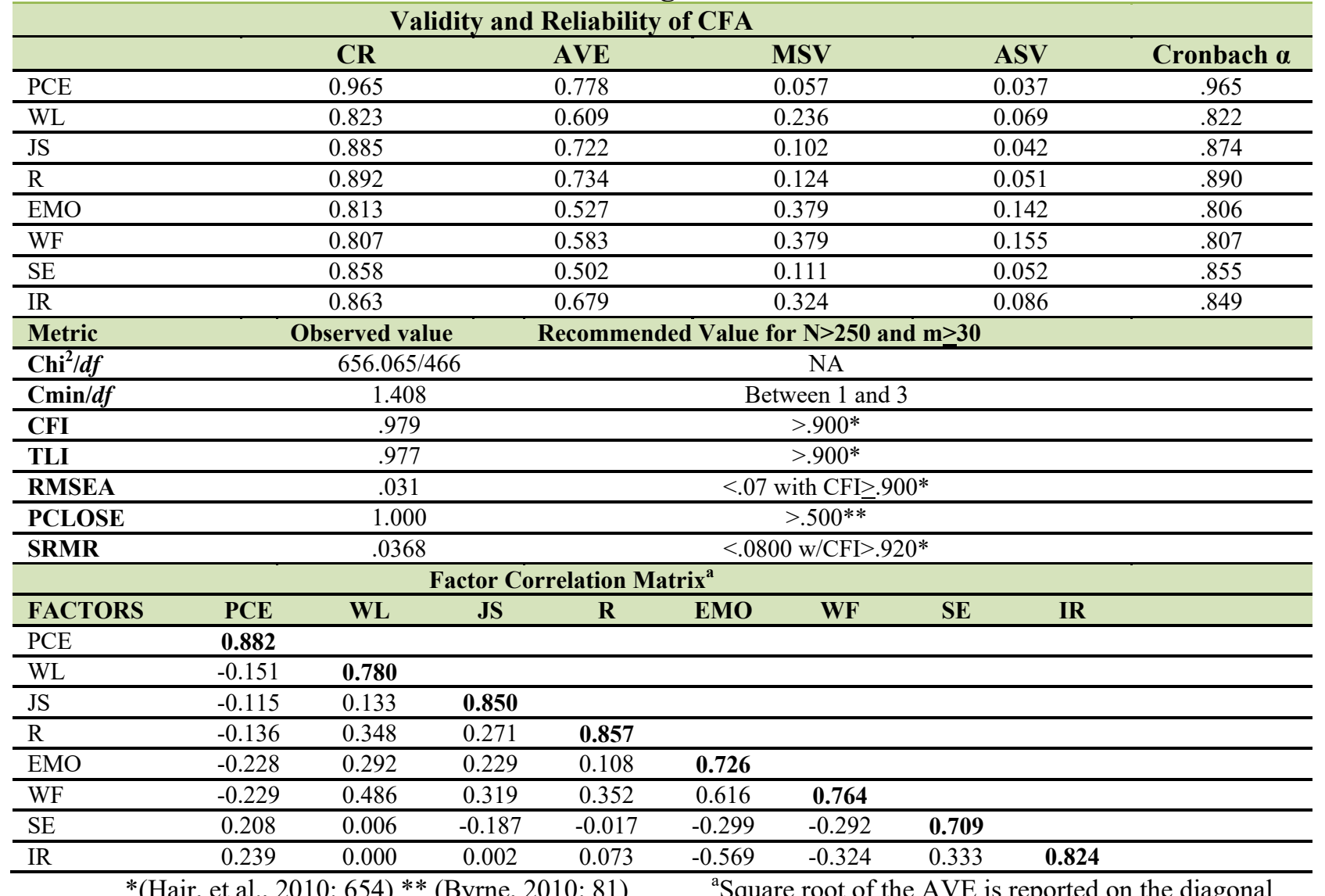


TABLE 4

\section{Comparison of $\mathbf{R}^{2}$ Statistic, Model Fit and Path Weights}

\begin{tabular}{|c|c|c|c|}
\hline \multicolumn{4}{|c|}{$\mathbf{R}^{2}$ Statistics and Model Fit } \\
\hline Metric & $\begin{array}{c}\begin{array}{c}\text { Proposed } \\
\text { Model }\end{array} \\
\end{array}$ & $\begin{array}{c}\text { Alternative } \\
\text { Model }\end{array}$ & $\begin{array}{c}\text { Recommended Value for } \\
\mathrm{N}>\mathbf{2 5 0} \text { and } \mathrm{m} \geq \mathbf{3 0}\end{array}$ \\
\hline $\mathbf{R}^{2}$ of Intentional Readiness & .233 & .428 & Not applicable \\
\hline $\mathrm{Chi}^{2} / \mathrm{df}$ & $1114.688 / 658$ & $1069.002 / 650$ & NA \\
\hline $\mathrm{Cmin} / \mathrm{df}$ & 1.694 & 1.645 & Between 1 and 3 \\
\hline CFI & .952 & .956 & $>.900 *$ \\
\hline TLI & .946 & .950 & $>.900$ \\
\hline RMSEA & .040 & .039 & $<.07$ with $\mathrm{CFI} \geq .900 *$ \\
\hline PCLOSE & 1.000 & 1.000 & $>.500 * *$ \\
\hline SRMR & .0747 & .0778 & $<.0800 \mathrm{w} / \mathrm{CFI}>.920^{*}$ \\
\hline
\end{tabular}

\begin{tabular}{|c|c|c|c|c|c|c|c|c|}
\hline \multicolumn{9}{|c|}{ Path Weights } \\
\hline \multirow[b]{2}{*}{ Path* } & \multicolumn{4}{|c|}{ Proposed Model } & \multicolumn{4}{|c|}{ Alternative Model } \\
\hline & $\begin{array}{l}\text { Std. } \\
\text { Est. }\end{array}$ & $\begin{array}{c}\text { Unstnd. } \\
\text { Est }\end{array}$ & $\begin{array}{l}\text { Std. } \\
\text { Error }\end{array}$ & P-Value & $\begin{array}{l}\text { Std. } \\
\text { Est. }\end{array}$ & $\begin{array}{c}\text { Unstnd. } \\
\text { Est }\end{array}$ & $\begin{array}{l}\text { Std. } \\
\text { Error }\end{array}$ & P-Value \\
\hline SE to IR & 0.205 & 0.277 & 0.080 & $<.001$ & 0.164 & 0.228 & 0.082 & 0.005 \\
\hline SE to PCE & 0.198 & 0.367 & 0.098 & $<.001$ & 0.201 & 0.371 & 0.098 & $<.001$ \\
\hline SE to D & -0.339 & -0.212 & 0.045 & $<.001$ & & & & \\
\hline SE to DS & & & & & -0.225 & -0.041 & 0.022 & 0.059 \\
\hline SE to DOS & & & & & -0.342 & -0.350 & 0.071 & $<.001$ \\
\hline PCE to IR & 0.137 & 0.099 & 0.036 & 0.006 & 0.127 & 0.095 & 0.034 & 0.005 \\
\hline D to IR & -0.320 & -0.691 & 0.156 & $<.001$ & & & & \\
\hline DS to IR & & & & & 0.177 & 1.358 & 0.660 & 0.039 \\
\hline DOS to IR & & & & & -0.555 & -0.751 & 0.101 & $<.001$ \\
\hline
\end{tabular}

* $\mathrm{SE}=$ self-efficacy; IR = intentional readiness for change; $\mathrm{PCE}=$ perceived collective efficacy; $\mathrm{D}=$ discomfort, a second order factor comprised of five dimensions; DS = discomfort from stretching, a second order factor comprised of three dimensions; DOS = discomfort from over-stretching, a second order factor comprised of two dimensions. 
FIGURE 1

SEM Results of Proposed Model

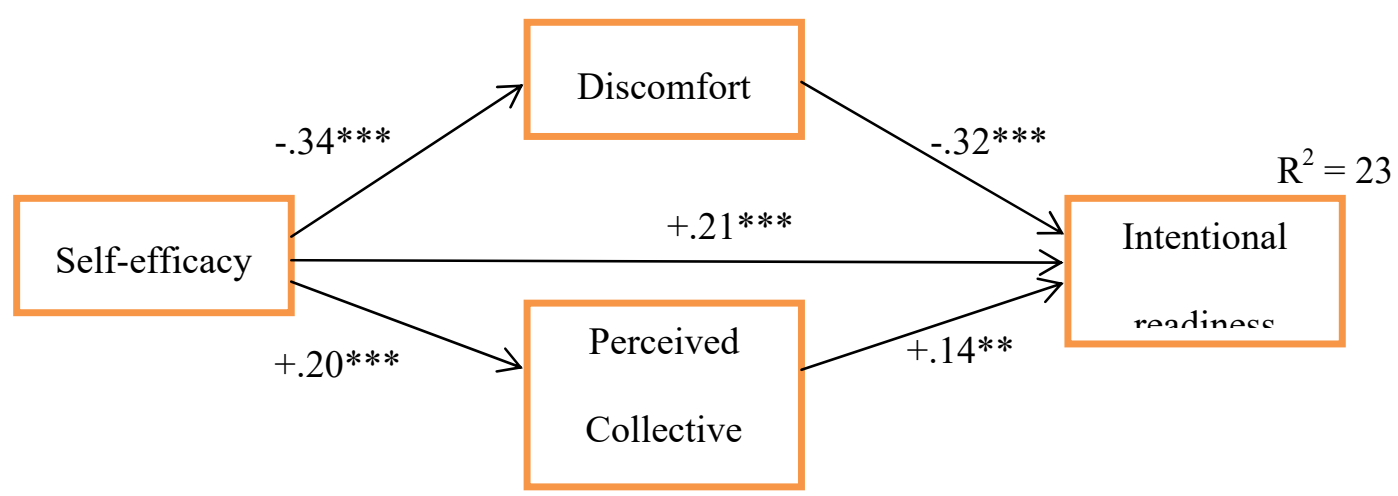

FIGURE 2

Alternative Model with Two Types of Discomfort

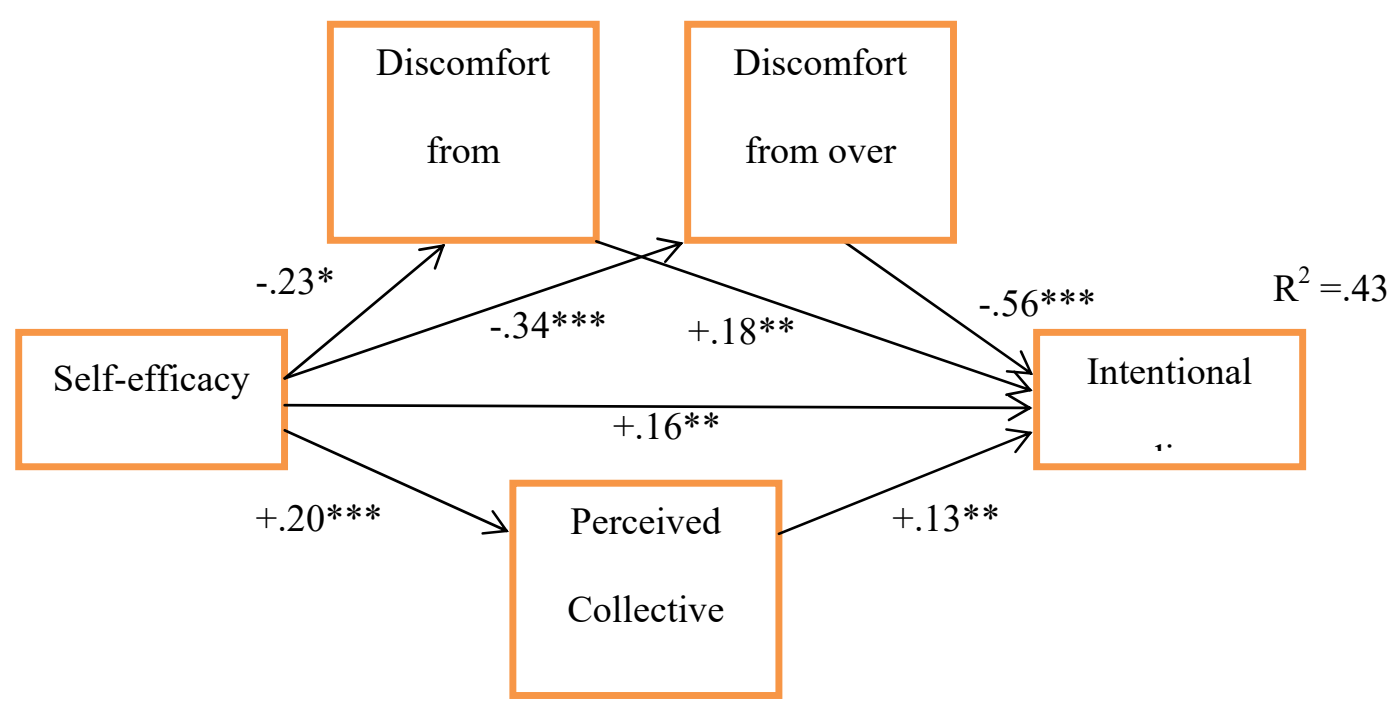

Note: $* * * \mathrm{p}<.001 ; * * \mathrm{p}<.05 ; * \mathrm{p}<.10$ 